BULLETIN OF THE POLISH

ACADEMY OF SCIENCES

MATHEMATICS

Vol. 65, No. 2, 2017

\title{
Multipeakons viewed as geodesics
}

by

\section{Tomasz CIEŚLAK, Michał GACZKOWSKI, Mariusz KUBKOWSKI and Marcin MAŁOGROSZ}

Presented by Piotr BILER

Summary. We adress the problem of qualitative properties of multipeakons, particular solutions of the Camassa-Holm equation. Our approach makes use of the well-known fact that the evolution of multipeakons is governed by the geodesic motion of a particle on an $N$-dimensional surface whose metric tensor is given via the inverse matrix to the one defining the Hamiltonian. Our approach yields some properties of twopeakons in a very simple way. We classify initial shapes of twopeakons according to the occurrence of collision. Moreover we extend the class of matrices that are invertible for similar reasons to the one occurring in the Hamiltonian. We get exact formulas for the inverses.

1. Introduction. We consider the motion of multipeakons as weak solutions of the Camassa-Holm equation. This motion is described via the Hamilton equations with the following (singular) Hamiltonian:

$$
H\left(p_{1}, \ldots, p_{n}, q_{1}, \ldots, q_{n}\right)=\frac{1}{2} \sum_{i, j=1}^{n} p_{i} p_{j} e^{-\left|q_{i}-q_{j}\right|}
$$

where $p_{i}, q_{i}$ are respectively amplitudes and positions of troughs and peaks of the multipeakon

$$
u(x, t)=\sum_{i=1}^{n} p_{i}(t) e^{-\left|x-q_{i}(t)\right|} .
$$

In other words, the Hamiltonian is given as

$$
H(p, q)=\frac{1}{2} p^{T} E(q) p,
$$

2010 Mathematics Subject Classification: 53A17, 15A09.

Key words and phrases: multipeakons, positive definite matrix, lagrangian.

Received 16 May 2017; revised 1 June 2017.

Published online 19 July 2017. 
where

$$
E(q)=\left(e^{-\left|q_{i}-q_{j}\right|}\right)_{i, j}
$$

is a symmetric matrix, and $p=\left(p_{1}, \ldots, p_{n}\right), q=\left(q_{1}, \ldots, q_{n}\right)$ are vectors in $\mathbb{R}^{n}$.

Multipeakons are important particular weak solutions of the CamassaHolm equation (see [5] and [11] for the global-in-time existence of weak solutions). Multipeakons have been extensively studied (see for instance [2], 4], [9], [10], 7]). As already noticed in [5], the positions $q_{i}(t)$ obey the geodesic equation with the metric tensor being the inverse of $E(q)$ given in (3). This is due to the fact that one can pass to the Lagrange description with the Lagrangian given by the Legendre transform of $H$ which is given by the bilinear form defined by $E^{-1}$ as long as it is invertible (see [1]). It is well-known that $H(q)$ is invertible if and only if $q_{i} \neq q_{j}$ for all $i \neq j$. Indeed, the Hamiltonian $H$ is actually given by

$$
2 H(p, q)=\frac{1}{2} \int_{-\infty}^{\infty}\left(u^{2}+u_{x}^{2}\right) d x,
$$

for $u$ as in (2). Formula (4) is a consequence of

$$
\begin{aligned}
\frac{1}{2} \int_{-\infty}^{\infty}\left(u^{2}+u_{x}^{2}\right) d x= & \frac{1}{2} \sum_{i, j=1}^{n} p_{i} p_{j} \int_{-\infty}^{\min \left\{q_{i}, q_{j}\right\}} e^{-\left|x-q_{i}\right|-\left|x-q_{j}\right|} d x \\
& +\frac{1}{2} \sum_{i, j=1}^{n} p_{i} p_{j} \int_{\max \left\{q_{i}, q_{j}\right\}}^{\infty} e^{-\left|x-q_{i}\right|-\left|x-q_{j}\right|} d x \\
= & \frac{1}{2} \sum_{i, j=1}^{n} 2 p_{i} p_{j} e^{\min \left\{q_{i}, q_{j}\right\}-\max \left\{q_{i}, q_{j}\right\}}=\sum_{i, j=1}^{n} p_{i} p_{j} e^{-\left|q_{i}-q_{j}\right|}
\end{aligned}
$$

as long as $q_{i} \neq q_{j}$. Hence, we see that $H(p, q) \geq 0$, with $H=0$ only if $u=0$. The latter is equivalent to $p_{1}=\cdots=p_{n}=0$. Thus $E$ is a positive definite (in particular invertible) matrix.

In the next section we give an alternative proof of this fact. As a consequence we shall see that a wider class of matrices containing $E$ as a special case is invertible. Moreover, we shall give a formula for the inverse of $E$. We shall use it to compute the Lagrangian associated to the Hamiltonian $H$.

In the last section we study the properties of twopeakons, interpreting them as trajectories of the Lagrange formulation of the problem. This allows us to arrive at very precise results concerning collisions of twopeakons.

Since multipeakons are solutions of a fully integrable Hamiltonian system before collision, much is known about their behavior (see for instance [5], [2, 9], 10]). However, the formulas obtained in those papers are rather 
complicated, so that listing the properties of solutions in the last section seems useful.

2. Invertibility of matrices and Lagrangian. There are many ways of demonstrating that $E$ is positive definite. One of them is presented in the Introduction. As noticed by the referee, another way would follow from the Bochner theorem. Indeed, the function $e^{-|x|}$ is the inverse Fourier transform of $\frac{1}{1+x^{2}}$ which is supported on the whole real line, so in particular $E$ is positive definite.

In this section we present an elementary probabilistic approach. The advantage of this method is that we directly obtain a formula for the inverse matrix. Moreover, in our approach the matrix $E$ is just a particular example of a more general class of matrices $A$ which are shown to be positive definite. The inverses of $A$ 's are found.

We start with the case where $q_{1}<\cdots<q_{n}$.

Lemma 2.1. Let $\rho_{1}, \ldots, \rho_{n-1} \in(-1,1)$. Let $A=\left[a_{i j}\right]$, where $a_{i i}=1$ for $i=1, \ldots, n$ and $a_{i j}=a_{j i}=\prod_{k=i}^{j-1} \rho_{k}$ for $1 \leq i<j \leq n$. Then $A$ is positive definite.

Proof. Let $\varepsilon_{1}, \ldots, \varepsilon_{n}$ be independent random variables such that $\mathbb{E} \varepsilon_{i}=0$ and $\mathbb{E} \varepsilon_{i}^{2}=1$ for $i=1, \ldots, n$. Let

$$
\left\{\begin{array}{l}
X_{1}=\varepsilon_{1}, \\
X_{i+1}=\rho_{i} X_{i}+\sqrt{1-\rho_{i}^{2}} \varepsilon_{i+1} \quad \text { for } i=1, \ldots, n-1 .
\end{array}\right.
$$

From the construction of $\mathbf{X}=\left(X_{1}, \ldots, X_{n}\right)$ we have by induction, for $i=$ $1, \ldots, n$,

$$
X_{i}=\sqrt{1-\rho_{i-1}^{2}} \varepsilon_{i}+\sum_{k=1}^{i-1} \sqrt{1-\rho_{k-1}^{2}} \prod_{j=k}^{i-1} \rho_{j} \varepsilon_{k} .
$$

From the above formula we can see that $X_{i}$ and $\varepsilon_{j}$ for $j>i$ are independent (by our choice of the vector $\varepsilon=\left(\varepsilon_{1}, \ldots, \varepsilon_{n}\right)$ ). Hence by induction $\operatorname{Var} X_{1}=$ $\operatorname{Var} \varepsilon_{1}=\mathbb{E} \varepsilon_{1}^{2}=1=a_{i i}$ and

$$
\begin{aligned}
\operatorname{Var} X_{i+1} & =\operatorname{Var}\left(\rho_{i} X_{i}+\sqrt{1-\rho_{i}^{2}} \varepsilon_{i+1}\right) \\
& =\rho_{i}^{2} \operatorname{Var} X_{i}+\left(1-\rho_{i}^{2}\right) \operatorname{Var} \varepsilon_{i+1}=\rho_{i}^{2} \operatorname{Var} X_{i}+\left(1-\rho_{i}^{2}\right)=1
\end{aligned}
$$

Moreover, we can prove by induction that for $j>i$,

$$
X_{j}=\prod_{k=i}^{j-1} \rho_{k} X_{i}+\sum_{k=i}^{j-1} \sqrt{1-\rho_{k}^{2}} \prod_{l=k}^{j-2} \rho_{l} \varepsilon_{k+1} .
$$


From this formula we obtain (for $i<j$ )

$$
\begin{gathered}
\operatorname{Cov}\left(X_{i}, X_{j}\right)=\operatorname{Cov}\left(X_{i}, \prod_{k=i}^{j-1} \rho_{k} X_{i}+\sum_{k=i}^{j-1} \sqrt{1-\rho_{k}^{2}} \prod_{l=k}^{j-2} \rho_{l} \varepsilon_{k+1}\right) \\
=\prod_{k=i}^{j-1} \rho_{k} \underbrace{\operatorname{Var} X_{i}}_{1}+\sum_{k=i}^{j-1} \sqrt{1-\rho_{k}^{2}} \prod_{l=k}^{j-2} \rho_{l} \underbrace{\operatorname{Cov}\left(X_{i}, \varepsilon_{k+1}\right)}_{0}=\prod_{k=i}^{j-1} \rho_{k}=a_{i j} .
\end{gathered}
$$

This means that $\operatorname{Var} \mathbf{X}=\mathbb{E} \mathbf{X X}^{T}=A$ and $A$ is non-negative definite, because for $v \in \mathbb{R}^{n}$ we obtain $v^{T} A v=\mathbb{E}\left\|v^{T} \mathbf{X}\right\|_{2}^{2} \geq 0$. To prove that $A$ is positive definite, observe that $\left(\rho_{0}=0\right)$

$$
\mathbf{X}=L_{1} \mathbf{X}+D \varepsilon,
$$

where

$$
L_{1}=\left[\begin{array}{cccc}
0 & \ldots & 0 & 0 \\
\rho_{1} & \ldots & 0 & 0 \\
\vdots & \ddots & \vdots & \vdots \\
0 & \ldots & \rho_{n-1} & 0
\end{array}\right]_{n \times n} \quad, \quad D=\left[\begin{array}{ccc}
\sqrt{1-\rho_{0}^{2}} & \cdots & 0 \\
\vdots & \ddots & \vdots \\
0 & \cdots & \sqrt{1-\rho_{n-1}^{2}}
\end{array}\right]_{n \times n} .
$$

Hence for $L:=\left(I-L_{1}\right)^{-1}$ we have $\mathbf{X}=L D \varepsilon$ and we see that

$$
A=\operatorname{Var} \mathbf{X}=L D \underbrace{\operatorname{Var} \varepsilon}_{I} D^{T} L^{T}=L D^{2} L^{T},
$$

so that $v^{T} A v=\left\|D L^{T} v\right\|_{2}^{2}>0$ for $v \in \mathbb{R}^{n} \backslash\{0\}$. It follows that $A$ is positive definite.

A particular case of the above lemma concerns matrices $E$ in the case of ordered $q_{i}$ 's.

LEMma 2.2. Let $v_{1}, \ldots, v_{n} \in \mathbb{R}$ with $v_{1}<\cdots<v_{n}$. Then the matrix $C=\left[c_{i j}\right] \in \mathbb{R}^{n \times n}$, where $c_{i j}=c_{j i}=e^{-v_{j}+v_{i}}$ for $j \geq i$, is positive definite.

Proof. Let $\rho_{i}=e^{-v_{i+1}+v_{i}}$ for $i=1, \ldots, n-1$. Then $c_{i j}=e^{-v_{j}+v_{i}}=$ $\prod_{k=i}^{j-1} \rho_{k}$ for $j \geq i$, and the matrix $C$ has the same form as $A$ in Lemma 2.1 . and the assumptions of that lemma are satisfied.

By a permutation of the random vector $\mathbf{X}$ we obtain the same result for any order of $q_{i}$ 's.

Lemma 2.3. Let $u_{1}, \ldots, u_{n} \in \mathbb{R}$ be pairwise distinct. Then the matrix $E=\left[e_{i j}\right]=\left[e^{-\left|u_{i}-u_{j}\right|}\right] \in \mathbb{R}^{n \times n}$ is positive definite.

Proof. Let $\pi:\{1, \ldots, n\} \rightarrow\{1, \ldots, n\}$ be a permutation such that $u_{\pi(i)}<u_{\pi(j)}$ for $i<j$. Let $v_{i}=u_{\pi(i)}$ for $i=1, \ldots, n$. Then the matrix $C$ defined as in Lemma 2.2 is positive definite and we can construct the 
vector $\mathbf{X}=\left(X_{1}, \ldots, X_{n}\right)$ as in Lemma 2.1 in such a way that $\operatorname{Cov}\left(X_{i}, X_{j}\right)=$ $c_{i j}=e^{-v_{j}+v_{i}}=e^{-\left|v_{j}-v_{i}\right|}$. Let $\mathbf{V}=\mathbf{X}_{\pi^{-1}}:=\left(X_{\pi^{-1}(1)}, \ldots, X_{\pi^{-1}(n)}\right)$. Then

$$
\operatorname{Cov}\left(V_{i}, V_{j}\right)=\operatorname{Cov}\left(X_{\pi^{-1}(i)}, X_{\pi^{-1}(j)}\right)=e^{-\left|v_{\pi^{-1}(i)}-v_{\pi^{-1}(j)}\right|}=e^{-\left|u_{i}-u_{j}\right|}=e_{i j}
$$

and from the definition of $\mathbf{V}$ we have $\mathbf{V}=P \mathbf{X}$, where $P=\left[p_{i j}\right] \in \mathbb{R}^{n \times n}$ and

$$
p_{i j}= \begin{cases}1, & j=\pi^{-1}(i), i=1, \ldots, n, \\ 0, & \text { otherwise. }\end{cases}
$$

Thus for every $b \in \mathbb{R}^{n} \backslash\{0\}$ we obtain

$$
b^{T} E b=b^{T} \operatorname{Var}(\mathbf{V}) b=b^{T} P C P^{T} b=d^{T} C d>0,
$$

where $d=P^{T} b \in \mathbb{R}^{n} \backslash\{0\}$. This ends our proof.

In the proof of Lemma 2.1, $A$ is defined as the covariance matrix of a random vector $\mathbf{X}$. This vector is defined in the inductive way, but we can also define it by means of a matrix equation. This is a key observation for obtaining the inverse matrix to $A$.

Corollary 2.1. $A^{-1}$ is the tridiagonal matrix

$$
\left[\begin{array}{ccccc}
a_{1} & b_{1} & 0 & \ldots & 0 \\
b_{1} & a_{2} & b_{2} & \ldots & 0 \\
0 & b_{2} & a_{3} & \ldots & 0 \\
\ldots & \ldots & \ldots \ldots \ldots & \ldots \\
0 & 0 & 0 & \ldots & a_{n}
\end{array}\right]
$$

where

$$
a_{i}=\frac{1}{1-\rho_{i-1}^{2}}+\frac{\rho_{i}^{2}}{1-\rho_{i}^{2}}, \quad b_{i}=\frac{-\rho_{i}}{1-\rho_{i}^{2}} .
$$

In particular, in the case of the matrix $E$ with $q_{1}<\cdots<q_{n}$ we have

$$
a_{i}=\frac{\sinh \left(q_{i+1}-q_{i-1}\right)}{2 \sinh \left(q_{i+1}-q_{i}\right) \sinh \left(q_{i}-q_{i-1}\right)}, \quad b_{i}=\frac{-1}{2 \sinh \left(q_{i+1}-q_{i}\right)} .
$$

Before proceeding with the proof let us remark that the matrix $E^{-1}$ was found in [7].

Proof. As noticed in (5), $X$ is given by

$$
X=L_{1} X+D \epsilon,
$$

where $L_{1}$ and $D$ are defined below (5). Hence

$$
\left(I-L_{1}\right) X=D \epsilon,
$$

and by taking the covariance matrix we obtain (see (6)

$$
\left(I-L_{1}\right) A\left(I-L_{1}\right)^{T}=D^{2} .
$$


Hence

$$
A^{-1}=\left(I-L_{1}\right)^{T} D^{-2}\left(I-L_{1}\right), \quad D^{-2}=\operatorname{diag}\left\{\frac{1}{1-\rho_{i-1}^{2}}\right\} .
$$

Thus we get

$$
\left(I-L_{1}\right)^{T} D^{-2}=\left[\begin{array}{ccccc}
1 & -\frac{\rho_{1}}{1-\rho_{1}^{2}} & \ldots & 0 & 0 \\
\cdots \ldots & \ldots \ldots & \ldots \ldots \ldots \ldots \ldots \ldots \ldots \\
0 & 0 & \ldots & \frac{1}{1-\rho_{n-2}^{2}} & -\frac{\rho_{n-1}}{1-\rho_{n-1}^{2}} \\
0 & 0 & \ldots & 0 & \frac{1}{1-\rho_{n-1}^{2}}
\end{array}\right],
$$

and multiplying the above matrix by $I-L_{1}$ on the right we obtain the claim of the corollary.

To obtain a simplified form of $a_{i}$ and $b_{i}$ in the most interesting case of the matrix $E$, we notice that on the one hand

$$
2 \sinh \left(q_{i+1}-q_{i}\right)=\frac{1-\left(e^{q_{i}-q_{i+1}}\right)^{2}}{e^{q_{i}-q_{i+1}}},
$$

and on the other

$$
\begin{aligned}
& \frac{e^{q_{i+1}-q_{i-1}}-e^{q_{i-1}-q_{i+1}}}{\left(e^{q_{i+1}-q_{i}}-e^{q_{i}-q_{i+1}}\right)\left(e^{q_{i}-q_{i-1}}-e^{q_{i-1}-q_{i}}\right)} \\
& =\frac{1-e^{2\left(q_{i-1}-q_{i+1}\right)}}{\left(1-e^{2\left(q_{i-1}-q_{i}\right)}\right)\left(1-e^{2\left(q_{i}-q_{i+1}\right)}\right)},
\end{aligned}
$$

where for $\rho_{i}:=e^{q_{i}-q_{i+1}}$ the last quantity is exactly

$$
\frac{1-\rho_{i-1}^{2} \rho_{i}^{2}}{\left(1-\rho_{i-1}^{2}\right)\left(1-\rho_{i}^{2}\right)}=\frac{1}{1-\rho_{i-1}^{2}}+\frac{\rho_{i}^{2}}{1-\rho_{i}^{2}}
$$

As long as multipeakons do not collide, the inverse matrix is used to compute the Lagrangian associated to the Hamiltonian $H$ by the Legendre transform,

$$
L(q, \dot{q})=\frac{1}{2} \dot{q}^{T} E^{-1}(q) \dot{q} .
$$

3. Equations of geodesics. Consider the $N$-peakon given by

$$
u(x, t):=\sum_{i=1}^{N} p_{i}(t) e^{-\left|x-q_{i}(t)\right|} .
$$

We can write the Hamiltonian $H$ in the form $H=\frac{1}{2} p^{T} E p$, where

$$
E=\left[e^{-\left|q_{i}-q_{j}\right|}\right]_{i, j=1}^{N} \text {. }
$$

As we already know, $E$ is strictly positive definite and invertible if $q_{i} \neq q_{j}$ for $i \neq j$. One can easily check that if $q_{i}=q_{j}$ for some $i \neq j$ then $E$ is singular. From the general theory we know that $q$ is described by a geodesic 
in the metric $E^{-1}$ wherever possible. In the case of $N=2$ we will write the equations of those geodesics. We start by considering a Riemannian manifold $\left(\mathbb{R}^{2}, g\right)$, where $g$ is of the form

$$
\frac{1}{1-e^{-2\left|q_{1}-q_{2}\right|}}\left[\begin{array}{ll}
1 & -e^{-\left|q_{1}-q_{2}\right|} \\
-e^{-\left|q_{1}-q_{2}\right|} & 1
\end{array}\right] .
$$

We look at this problem in the coordinates

$$
s_{1}:=\frac{q_{1}+q_{2}}{2}, \quad s_{2}:=\frac{q_{1}-q_{2}}{2} .
$$

THEOREM 3.1. The equation for the geodesics in $\left(s_{1}, s_{2}\right)$ coordinates is

$$
\left\{\begin{array}{l}
\dot{s}_{1}=z_{1}, \\
\dot{s}_{2}=z_{2}, \\
\dot{z}_{1}=-\operatorname{sgn} s_{2} \frac{2 e^{-2\left|s_{2}\right|}}{1+e^{-2\left|s_{2}\right|}} z_{1} z_{2}, \\
\dot{z}_{2}=\operatorname{sgn} s_{2} \frac{e^{-2\left|s_{2}\right|}\left(1-e^{-2\left|s_{2}\right|}\right)}{\left(1+e^{-2\left|s_{2}\right|}\right)^{2}} z_{1}^{2}+\operatorname{sgn} s_{2} \frac{e^{-2\left|s_{2}\right|}}{1-e^{-2\left|s_{2}\right|}} z_{2}^{2} .
\end{array}\right.
$$

Proof. In the new coordinates the tensor metric has the form

$$
\begin{aligned}
g & =\frac{1}{1-e^{-2\left|q_{1}-q_{2}\right|}}\left(d q_{1} \otimes d q_{1}-e^{-\left|q_{1}-q_{2}\right|} d q_{1} \otimes d q_{2}\right. \\
& \left.-e^{-\left|q_{1}-q_{2}\right|} d q_{2} \otimes d q_{1}+d q_{2} \otimes d q_{2}\right) \\
& =\frac{1}{1-e^{-4\left|s_{2}\right|}}\left(2\left(1-e^{-2\left|s_{2}\right|}\right) d s_{1} \otimes d s_{1}+2\left(1+e^{-2\left|s_{2}\right|}\right) d s_{2} \otimes d s_{2}\right) \\
& =\frac{2}{1+e^{-2\left|s_{2}\right|}} d s_{1} \otimes d s_{1}+\frac{2}{1-e^{-2\left|s_{2}\right|}} d s_{2} \otimes d s_{2} .
\end{aligned}
$$

And the matrix of $g$ is

$$
\left[\begin{array}{cc}
\frac{2}{1+e^{-2\left|s_{2}\right|}} & 0 \\
0 & \frac{2}{1-e^{-2\left|s_{2}\right|}}
\end{array}\right] .
$$

Observe that Christoffel's symbols are easy to compute: First,

$$
\Gamma_{11}^{1}=\Gamma_{22}^{1}=\Gamma_{12}^{2}=0 .
$$

Since

$$
\frac{\partial g_{11}}{\partial s_{2}}=\operatorname{sgn} s_{2} \frac{4 e^{-2\left|s_{2}\right|}}{\left(1+e^{-2\left|s_{2}\right|}\right)^{2}}, \quad \frac{\partial g_{22}}{\partial s_{2}}=-\operatorname{sgn} s_{2} \frac{4 e^{-2\left|s_{2}\right|}}{\left(1-e^{-2\left|s_{2}\right|}\right)^{2}},
$$


the other symbols are

$$
\begin{aligned}
& \Gamma_{12}^{1}=\frac{\partial g_{11}}{\partial s_{2}} \frac{1+e^{-2\left|s_{2}\right|}}{4}=\operatorname{sgn} s_{2} \frac{e^{-2\left|s_{2}\right|}}{1+e^{-2\left|s_{2}\right|}}, \\
& \Gamma_{11}^{2}=-\frac{\partial g_{11}}{\partial s_{2}} \frac{1-e^{-2\left|s_{2}\right|}}{4}=-\operatorname{sgn} s_{2} \frac{e^{-2\left|s_{2}\right|}\left(1-e^{-2\left|s_{2}\right|}\right)}{\left(1+e^{-2\left|s_{2}\right|}\right)^{2}}, \\
& \Gamma_{22}^{2}=\frac{\partial g_{22}}{\partial s_{2}} \frac{1-e^{-2\left|s_{2}\right|}}{4}=-\operatorname{sgn} s_{2} \frac{e^{-2\left|s_{2}\right|}}{1-e^{-2\left|s_{2}\right|}} .
\end{aligned}
$$

Hence, we can write the equations of the geodesics in the form (9).

Analysis of (9) gives us some insight into when twopeakons can collide. Let us start with the following observation.

LEMMA 3.1. If

$$
\left(q_{1}\left(t_{0}\right)-q_{2}\left(t_{0}\right)\right)\left(p_{1}\left(t_{0}\right)-p_{2}\left(t_{0}\right)\right)>0,
$$

then there is no collision.

Proof. Observe that $\dot{z}_{2}$ always has the same sign as $s_{2}$. So in case $s_{2}>0$ we see that if $z_{2}=\alpha>0$ then $z_{2}$ is always greater than $\alpha$. From the equation we see that $\dot{s}_{2}=z_{2} \geq \alpha$ and a collision cannot occur. The same happens if $s_{2}<0$ and $z_{2}<0$.

Let $\left(q_{1}, q_{2}\right)$ describe the geodesic in the previous coordinates. Our considerations show that if

$$
\left(q_{1}\left(t_{0}\right)-q_{2}\left(t_{0}\right)\right)\left(\dot{q}_{1}\left(t_{0}\right)-\dot{q}_{2}\left(t_{0}\right)\right)>0,
$$

then there is no collision.

Moreover, with the Hamiltonian $H$ one can associate the Lagrangian $L$ given by

$$
L(q, \dot{q})=\frac{1}{2\left(1-e^{-2\left|q_{1}-q_{2}\right|}\right)} \dot{q}^{T}\left[\begin{array}{cc}
1 & -e^{-\left|q_{1}-q_{2}\right|} \\
-e^{-\left|q_{1}-q_{2}\right|} & 1
\end{array}\right] \dot{q} .
$$

Using $L$ we can obtain $p$ from $\dot{q}$ via

$$
p_{i}=\frac{\partial L}{\partial \dot{q}_{i}} .
$$

This gives

$$
\begin{aligned}
& p_{1}=\frac{1}{1-e^{-2\left|q_{1}-q_{2}\right|}}\left(\dot{q}_{1}-e^{-\left|q_{1}-q_{2}\right|} \dot{q}_{2}\right), \\
& p_{2}=\frac{1}{1-e^{-2\left|q_{1}-q_{2}\right|}}\left(\dot{q}_{2}-e^{-\left|q_{1}-q_{2}\right|} \dot{q}_{1}\right) .
\end{aligned}
$$

Since

$$
p_{1}-p_{2}=\frac{\dot{q}_{1}-\dot{q}_{2}}{1-e^{-\left|q_{1}-q_{2}\right|}},
$$


(10) implies

$$
\left(q_{1}\left(t_{0}\right)-q_{2}\left(t_{0}\right)\right)\left(p_{1}\left(t_{0}\right)-p_{2}\left(t_{0}\right)\right)>0 .
$$

Using the equations for the geodesics one can also study the well-known peakon-antipeakon case.

Lemma 3.2. Let $p_{1}+p_{2}=0$ and $q_{1}>q_{2}, p_{2}>p_{1}$. Then a collision occurs in finite time.

Proof. Now suppose that in $(9), z_{1}=0, s_{2}>0$ and $z_{2}<0$. Then

$$
\left\{\begin{array}{l}
\dot{s}_{2}=z_{2}, \\
\dot{z}_{2}=\frac{e^{-2 s_{2}}}{1-e^{-2 s_{2}}} z_{2}^{2} .
\end{array}\right.
$$

The assumption $z_{1}=0$ implies that $\dot{q}_{1}+\dot{q}_{2}=0$. In terms of $p$ we can rewrite this as

$$
p_{1}+p_{2}=\frac{\dot{q}_{1}+\dot{q}_{2}}{1+e^{-\left|q_{1}-q_{2}\right|}}=0 .
$$

One can check that

$$
z_{2}=C \sqrt{1-e^{-2 s_{2}}}
$$

for $C<0$. Now it is enough to solve

$$
\dot{s}_{2}=C \sqrt{1-e^{-2 s_{2}}} .
$$

The solution is

$$
s_{2}=-\frac{1}{2} \ln \left(1-\left(\frac{D e^{2 C t}-1}{D e^{2 C t}+1}\right)^{2}\right),
$$

where $D>0$. Hence for $t_{1}=-\frac{\ln D}{2 C}$ we see that $s_{2}=0$.

Using the equations of geodesics one can determine whether there is a collision in the general case. However, this requires some more advanced tools of differential geometry and will be given elsewhere, together with the analysis of prolongation of solutions past a collision. In [8], exact formulas for twopeakon solutions are given; one can analyze the question of collision using them. Below we fully classify the initial conditions from the point of view of collisions.

TheOREM 3.2. Let $q_{1}(0)<q_{2}(0)$. Then a collision occurs if and only if $p_{1}(0)>0>p_{2}(0)$. Moreover, the time of collision $T^{*}$ is given by

$$
T^{*}=\frac{1}{a} \ln \left(\frac{p_{2}(0)-p_{1}(0)-a}{p_{2}(0)-p_{1}(0)+a}\right),
$$

where $a=\sqrt{\left(p_{2}(0)-p_{1}(0)\right)^{2}+4 p_{1}(0) p_{2}(0) e^{-\left(q_{2}(0)-q_{1}(0)\right)}}$. 
Proof. Recall the Hamiltonian system

$$
\left\{\begin{array}{l}
\dot{p}_{1}=-p_{1} p_{2} e^{-\left|q_{2}-q_{1}\right|} \\
\dot{p}_{2}=p_{1} p_{2} e^{-\left|q_{2}-q_{1}\right|} \\
\dot{q}_{1}=p_{1}+p_{2} e^{-\left|q_{2}-q_{1}\right|} \\
\dot{q}_{2}=p_{2}+p_{1} e^{-\left|q_{2}-q_{1}\right|}
\end{array}\right.
$$

and its first integrals

$$
H_{0}=p_{1}+p_{2}, \quad H_{1}=\frac{1}{2}\left(p_{1}^{2}+p_{2}^{2}\right)+p_{1} p_{2} e^{-\left|q_{2}-q_{1}\right|} .
$$

Since $q_{1}(0)<q_{2}(0)$, until the collision we have $q_{1}(t)<q_{2}(t)$ and we may drop the absolute value in the above equations. In the new variables,

$$
x=e^{-\left(q_{2}-q_{1}\right)}, \quad y=p_{2}-p_{1},
$$

the system under consideration is

$$
\begin{aligned}
\dot{y} & =\dot{p}_{2}-\dot{p}_{1}=2 p_{1} p_{2} e^{-\left(q_{2}-q_{1}\right)} \\
& =\frac{1}{2}\left(\left(p_{1}+p_{2}\right)^{2}-\left(p_{1}-p_{2}\right)^{2}\right) x=\frac{1}{2}\left(H_{0}^{2}-y^{2}\right) x, \\
\dot{x} & =\left(\dot{q}_{1}-\dot{q}_{2}\right) e^{-\left(q_{2}-q_{1}\right)}=\left(p_{1}+p_{2} e^{-\left(q_{2}-q_{1}\right)}-p_{2}-p_{1} e^{-\left(q_{2}-q_{1}\right)}\right) x \\
& =\left(p_{1}-p_{2}\right)\left(1-e^{-\left(q_{2}-q_{1}\right)}\right) x=-x(1-x) y .
\end{aligned}
$$

Moreover we have

$$
\begin{aligned}
H_{1} & =\frac{1}{2}\left(p_{1}^{2}+p_{2}^{2}\right)+p_{1} p_{2} e^{-\left(q_{2}-q_{1}\right)} \\
& =\frac{1}{4}\left(\left(p_{1}+p_{2}\right)^{2}+\left(p_{1}-p_{2}\right)^{2}\right)+\frac{1}{4}\left(\left(p_{1}+p_{2}\right)^{2}-\left(p_{1}-p_{2}\right)^{2}\right) x \\
& =\frac{1}{4}\left(H_{0}^{2}+y^{2}+\left(H_{0}^{2}-y^{2}\right) x\right) .
\end{aligned}
$$

Hence

$$
\left(H_{0}^{2}-y^{2}\right) x=4 H_{1}-H_{0}^{2}-y^{2}
$$

Because

$$
4 H_{1}-H_{0}^{2}=H_{0}^{2} x+y^{2}(1-x)>0
$$

we may denote $a^{2}=4 H_{1}-H_{0}^{2}$ and find that

$$
\dot{y}=\frac{1}{2}\left(a^{2}-y^{2}\right) .
$$

After integration we obtain

$$
\left|\frac{y(t)+a}{y(t)-a}\right|=e^{a t}\left|\frac{y(0)+a}{y(0)-a}\right|,
$$

and finally the solution is

$$
y(t)=a \frac{(a+y(0)) e^{a t}-(a-y(0))}{(a+y(0)) e^{a t}+(a-y(0))} .
$$


The blow-up occurs if and only if the denominator vanishes, and the blow-up time is given by

$$
T^{*}=\frac{1}{a} \ln \left(\frac{y(0)-a}{y(0)+a}\right)=\frac{1}{a} \ln \left(\frac{p_{2}(0)-p_{1}(0)-a}{p_{2}(0)-p_{1}(0)+a}\right) .
$$

Observe that $T^{*}>0$ if and only if $\frac{y(0)-a}{y(0)+a}>1$, which is equivalent to $y(0)<$ $-a$. The last inequality is equivalent to the system of inequalities

Observe that

$$
\left\{\begin{array}{l}
y(0)<0 \\
y(0)^{2}>a^{2}
\end{array}\right.
$$

$$
\begin{aligned}
y(0)^{2}>a^{2} & \Leftrightarrow\left(p_{1}(0)-p_{2}(0)\right)^{2}>x\left(p_{1}(0)+p_{2}(0)\right)^{2}+(1-x)\left(p_{1}(0)-p_{2}(0)\right)^{2} \\
& \Leftrightarrow-2 p_{1}(0) p_{2}(0)>2 p_{1}(0) p_{2}(0)(x-(1-x)) \\
& \Leftrightarrow 0>p_{1}(0) p_{2}(0) .
\end{aligned}
$$

Finally, the condition for blow-up is

which is equivalent to

$$
\left\{\begin{array}{l}
p_{2}(0)-p_{1}(0)<0 \\
p_{1}(0) p_{2}(0)<0
\end{array}\right.
$$

$$
p_{1}(0)>0>p_{2}(0) \text {. }
$$

In Lagrangian coordinates the collision condition can be expressed as follows:

REMARK 3.1. If $q_{1}(0)<q_{2}(0)$ then collision occurs if and only if one of the following conditions is satisfied:

- $\dot{q}_{1}(0) \geq 0, \dot{q}_{2}(0)<0$,

- $\dot{q}_{1}(0)>0, \dot{q}_{2}(0) \leq 0$,

- $\dot{q}_{1}(0)>0, \dot{q}_{2}(0)>0$ and

$$
\frac{\dot{q}_{2}(0)}{\dot{q}_{1}(0)}<e^{-\left(q_{2}(0)-q_{1}(0)\right)}
$$

- $\dot{q}_{1}(0)<0, \dot{q}_{2}(0)<0$ and

$$
\frac{\dot{q}_{2}(0)}{\dot{q}_{1}(0)}>e^{q_{2}(0)-q_{1}(0)} .
$$

Proof. Since

$$
\left[\begin{array}{c}
p_{1} \\
p_{2}
\end{array}\right]=\frac{1}{1-e^{-2\left(q_{2}-q_{1}\right)}}\left[\begin{array}{cc}
1 & -e^{-\left(q_{2}-q_{1}\right)} \\
e^{-\left(q_{2}-q_{1}\right)} & 1
\end{array}\right]\left[\begin{array}{c}
\dot{q}_{1} \\
\dot{q}_{2}
\end{array}\right],
$$

the collision condition obtained in Theorem 3.2 can be formulated as

$$
\left\{\begin{array}{l}
\dot{q}_{1}(0)-e^{-\left(q_{2}(0)-q_{1}(0)\right)} \dot{q}_{2}(0)>0, \\
-e^{-\left(q_{2}(0)-q_{1}(0)\right)} \dot{q}_{1}(0)+\dot{q}_{2}(0)<0,
\end{array}\right.
$$

from which the claim follows. 
Acknowledgements. T. C. was partially supported by National Science Centre under grant number 2013/09/D/ST1/03687.

\section{References}

[1] V. I. Arnold, Mathematical Methods of Classical Mechanics, 2nd ed., Grad. Texts in Math. 60, Springer, 1989.

[2] R. Beals, D. H. Sattinger and J. Szmigielski, Multipeakons and the classical moment problem, Adv. Math. 140 (1998), 190-206.

[3] P. Billingsley, Probability and Measure, Wiley, 1979.

[4] A. Bressan and A. Constantin, Global dissipative solutions of the Camassa-Holm equation, Anal. Appl. (Singapore) 5 (2007), 1-27.

[5] R. Camassa and D. Holm, An integrable shallow water equation with peaked solitons, Phys. Rev. Lett. 71 (1993), 1661-1664.

[6] A. Constantin and J. Escher, Global existence and blow-up for a shallow water equation, Ann. Scuola Norm. Sup. Pisa Cl. Sci. 26 (1998), 303-328.

[7] J. Eckhardt and A. Kostenko, An isospectral problem for global conservative multipeakon solutions of the Camassa-Holm equation, Comm. Math. Phys. 329 (2014), 893-918.

[8] K. Grunert and H. Holden, The general peakon-antipeakon solution for the CamassaHolm equation, J. Hyperbolic Differerential Equations 13 (2016), 353-380.

[9] H. Holden and X. Raynaud, Global dissipative multipeakon solutions of the CamassaHolm equation, Comm. Partial Differerential Equations 33 (2008), 2040-2063.

[10] H. Holden and X. Raynaud, Global conservative multipeakon solutions of the Camassa-Holm equation, J. Hyperbolic Differerential Equations 4 (2007), 39-64.

[11] Z. Xin and P. Zhang, On the weak solutions to a shallow water equation, Comm. Pure Appl. Math. 53 (2000), 1411-1433.

Tomasz Cieślak, Michał Gaczkowski,

Mariusz Kubkowski

Marcin Małogrosz

Institute of Mathematics

Polish Academy of Sciences

00-656 Warszawa, Poland

E-mail: cieslak@impan.pl

m.gaczkowski@impan.pl

mmalogrosz@impan.pl
Wydział Matematyki i Nauk Informacyjnych

Politechnika Warszawska

00-662 Warszawa, Poland

E-mail: M.Kubkowski@mini.pw.edu.pl 